\title{
Carriage Rate of Group B Streptococci in Pregnant Women in Three Teaching Hospitals in Shiraz, Iran
}

\author{
Parvin Hassanzadeh ${ }^{a}$ Mohammad Motamedifar ${ }^{b, c}$ Maral Namdari Gharaghani ${ }^{b}$ \\ ${ }^{a}$ Department of Biology, School of Science, Shiraz University, ${ }^{b}$ Department of Bacteriology and Virology, \\ Medical School, and 'Shiraz HIV/Aids Research Center, Shiraz University of Medical Sciences, Shiraz, Iran
}

\section{Key Words}

Carriage rate - Group B streptococci • Pregnant women •

Shiraz $\cdot$ Iran

\begin{abstract}
Objective: The main goal of this study was to assess differences in the vaginal flora of pregnant women and provide a detailed evaluation of vaginal swabs for the presence of group B streptococcus (GBS) and other organisms in three teaching hospitals in Shiraz, southwest of Iran. Subjects and Methods: In a cross-sectional study from April 2006 to March 2007,310 women with vaginal delivery at three obstetric wards of teaching hospitals in Shiraz were studied. Swab specimens from the vagina and rectum were collected and were examined bacteriologically. All of the newborns were followed up for 3 months. Results: Of 310 pregnant women, $43(13.8 \%)$ were colonized with GBS. There were no differences between GBS carriers and GBS-negative women in risk factors like preterm rupture of membranes $(p=0.77)$ and preterm labor $(p=0.53)$. One neonate was hospitalized due to meningitis and GBS was detected in CSF culture and Gram staining. Conclusion: Our data showed that the prevalence of GBS carriage rate in pregnant women in Shiraz, Iran was high. The poor correlation between risk factors and GBS carriage requires further investigation in larger groups.
\end{abstract}

Copyright $\odot 2011$ S. Karger AG, Basel
(C) 2011 S. Karger AG, Basel

1011-7571/11/0203-0277\$38.00/0

Fax +4161306 1234

E-Mail karger@karger.ch

www.karger.com
Accessible online at: www.karger.com/mpp

\section{Introduction}

Streptococcus agalactiae, belonging to group B streptococci (GBS), emerged during the 1960s as an important cause of neonatal disease, and by the 1970s, it was already established as a leading cause of infections in the newborn [1,2]. Also GBS is an important agent of maternal infections, such as chorioamnionitis, endometritis, urinary tract and surgical-site infections [3-5]. Two forms of GBS disease in infants are well recognized: early- and late-onset disease. Early-onset disease is defined as isolation of GBS from a normally sterile site (i.e., blood and/or cerebrospinal fluid) in an infant less than 7 days of age with clinical symptoms and signs compatible with a systemic infection. It accounts for $80-85 \%$ of neonatal infections, has a higher mortality rate and it is acquired through vertical transmission from colonized mothers [1, $5,6]$. Exposure of the neonate to the organism occurs either by an ascending route in utero through ruptured or intact membranes or by acquisition during passage through the birth canal. The three most common clinical presentations include sepsis, pneumonia, and meningitis [5].

Late-onset disease usually occurs in infants between 1 week and up to 3 months of age with meningitis being the most common clinical presentation $[5,7]$. The source of bacterial strains causing late-onset disease is less well

M. Motamedifar

Department of Bacteriology and Virology

Shiraz Medical School/Shiraz HIV/Aids Research Center (SHARC)

Shiraz University of Medical Sciences, PO Box 71455-119, Shiraz (Iran)

Tel./Fax +98 711228 0916, E-Mail motamedm@sums.ac.ir 
understood and may involve community or nosocomial acquisition, although there is also evidence that in some infants with late-onset disease, the GBS causing the infection shares the same serotype as the GBS isolated from their mothers, suggesting a maternal source $[5,8]$.

Results collected from different geographic regions indicate a heterogeneous prevalence. Rates of colonization may vary greatly according to the sample collection site, the culture medium used, the ethnic group, geographical location, socioeconomic status, immunological factors and the age of the population investigated $[9,10]$.

According to reports from Western countries, especially the United States, vaginal colonization occurs in $11-30 \%$ of all pregnant women, and $50-75 \%$ of their infants become colonized on their mucosal surfaces and on skin $[7,11,12]$. The frequency of maternal carriage in vagina has been reported from some developing countries, including Kuwait: 14.6\% [13], India: 5.8\%; Libya: 5\%; Saudi Arabia: $13.9 \%$ [14], and Brazil: 14.6\% [9].

With the objective of reducing neonatal morbidity and mortality due to GBS, different countries have implemented strategies of intrapartum prophylactic antibiotic therapy, resulting in a significant decrease in the incidence of neonatal infection from 2.7 to 0.4 per 1,000 live births $[9,15]$. Strategies involve antenatal screening to detect colonization or treatment of women with risk factors such as prolonged rupture of membranes, intrapartum fever, preterm labor and history of maternal colonization during pregnancy which reflects in part the burden of GBS. The Centers for Disease Control (CDC) recommend either risk assessment or screening for group B streptococcal colonization in pregnant women to identify candidates for intrapartum prophylaxis. Screening consists of obtaining vaginal and anal specimens for culture at 35-37 weeks' gestation. Women can be negative in the first 30 weeks and then become positive in the last 10 weeks. Those who are positive in the last trimester could be negative in the first or second [15]. In Iran, there is no uniform policy and many institutions do not adhere strictly to the CDC guidelines. Furthermore, there are few publications on this subject, and some authors have found a GBS isolation rate of $5-25 \%$ among Iranian parturient women [16, 17]. Herein, we report the colonization rate of GBS in pregnant women and their neonates and some risk factors in the city Shiraz, Southwest of Iran in order to adopt a preventive strategy for decreasing probable invasive infections in neonates [18].

\section{Subjects and Methods}

\section{Study Design}

This study was conducted from April 2006 to March 2007 at three obstetric teaching hospitals (Zeinabie, Shooshtari and Shiraz). During the study period, none of the participating hospitals had a written policy for prevention of GBS disease through intrapartum chemoprophylaxis. Three hundred and ten women were studied over a 1-year period (2006-2007), of whom 137 were from Shiraz Hospital, 107 from Zeinabie Hospital and the remaining 66 from the Shooshtari Hospital. Mean age was $25.6 \pm 5.4$ years (20.2-31.0). The study was approved by the Research Ethics Committee, Shiraz University of Medical Sciences, and all participating women gave written informed consent.

\section{Culture and Identification of GBS}

The specimens of 310 pregnant women with gestational age of 20 weeks or more who presented to the labor and delivery room were cultured for GBS. A single vaginorectal swab for culture of GBS was taken from participants. All samples were taken before conducting a pelvic examination and before using any disinfecting materials. The swab was first inserted into the vagina (without using a speculum). For vaginal specimens, excessive secretions or discharge was wiped out, and secretions from the mucosa of the lower third of the vagina were obtained with a swab. Afterwards, a swab was carefully inserted approximately $2.5 \mathrm{~cm}$ beyond the anal sphincter and then gently rotated to touch anal crypts.

Swabs were immediately placed in selective enrichment Todd-Hewitt broth with gentamicin $(8 \mathrm{mg} / \mathrm{l})$ and nalidixic acid $(15 \mathrm{mg} / \mathrm{l})$, as recommended by the CDC, and sent to the Laboratory of Microbiology. At the laboratory, samples were incubated for $24 \mathrm{~h}$ at $37^{\circ} \mathrm{C}$ in a $5 \% \mathrm{CO}_{2}$ atmosphere. Colonies of beta-hemolytic streptococci and Gram-positive diplococci were plated onto blood agar and incubated for 24-48 h, after which they were identified by the negative catalase test process. After obtaining a purified GBS culture, identified both by the presence of hemolysis and negative catalase, colonization was further confirmed by two confirmatory tests: the CAMP and the positive latex agglutination tests [19]. All of the samples also underwent routine microbiological culture and bacterial isolates were identified by using Gram staining and standard biochemical methods. Chlamydia, viruses, and T-strain mycoplasma and Trichomonas were not sought in this study, and only simple methods of anaerobic culture were used.

After labor and delivery, all women underwent a bedside postpartum interview using a specific uniform questionnaire. Maternal demographic status was assessed, including age, nationality and origin. Maternal general health variables included verified disease or infections in present and/or past gestations, antibiotic treatment in current pregnancy; obstetric status variables included parity and the number of gestations, past abortions, number of fetuses in the current pregnancy, gestational week of membrane rupture, mode of current labor, history of cesarean sections or premature deliveries and type of contraception used before pregnancy. The medical records of all neonates born to maternal carriers were examined after 12 weeks for neonatal hospitalizations and GBS diseases (meningitis, sepsis). 
Table 1. Age, ethnicity, residence, number of pregnancies and contraception method recorded for 310 pregnant women from Shiraz, Iran

\begin{tabular}{lccl}
\hline $\begin{array}{l}\text { Epidemiological } \\
\text { information }\end{array}$ & $\begin{array}{l}\text { GBS carrier } \\
\mathrm{n}(\%)\end{array}$ & $\begin{array}{l}\text { GBS-negative } \\
\mathrm{n}(\%)\end{array}$ & $\mathrm{p}$ value \\
\hline $\begin{array}{l}\text { Age, years } \\
<20\end{array}$ & $7(16.3)$ & $27(10.1)$ & 0.98 \\
$20-34$ & $32(74.4)$ & $220(82.4)$ & 0.65 \\
$\quad>34$ & $4(9.3)$ & $20(7.5)$ & 0.48 \\
Ethnic origin & & & \\
$\quad$ Iranian & $38(88.4)$ & $216(80.9)$ & 0.35 \\
$\quad$ Afghani & $5(11.6)$ & $51(19.1)$ & \\
Pregnancy & & & \\
$\quad$ Primiparous & $21(48.8)$ & $122(45.7)$ & 0.21 \\
$\quad$ Multiparous & $22(51.2)$ & $145(54.3)$ & \\
Residence & & & \\
$\quad$ Urban & $31(72.1)$ & $163(61.1)$ & 0.42 \\
$\quad$ Rural & $12(27.9)$ & $104(38.9)$ & \\
Contraception & & & \\
$\quad$ Hormonal & $8(18.6)$ & $104(38.9)$ & 0.019 \\
$\quad$ Intrauterine & $5(11.6)$ & $38(14.2)$ & 0.62 \\
$\quad$ Barrier methods & $2(4.7)$ & $42(15.8)$ & 0.017 \\
$\quad$ None & $28(65.1)$ & $83(31.1)$ & \\
Previous abortion history & & & \\
$\quad$ Yes & $4(9.3)$ & $21(7.9)$ & - \\
$\quad$ No & $39(90.7)$ & $246(92.1)$ & \\
\hline & & & \\
\hline
\end{tabular}

Statistical Analyses

SPSS software version 15 was used to analyze the data and the $\chi^{2}$ test for pair differences was done. Descriptive summarization of data consisted of frequency counts and percentages. Fisher's exact test was used for noncategorical variables; $\mathrm{p}<0.05$ was considered to be significant.

\section{Results}

\section{Epidemiological and Clinical Information}

Of the 310 women, 43 (13.8\%) were colonized by GBS. There was no significant difference between carriage rates at the three hospitals $(p=0.366)$. None of the women reported having had more than one sexual partner in the previous 12 months; most $(254,81.9 \%)$ were Iranians living in urban areas (62.6\%) and used hormonal contraception (36.1\%) before pregnancy. Further epidemiological data are given in table 1.

With respect to the obstetric characteristics, mean gestational age was $38.3 \pm 3.3$ weeks; 151 (48.8\%) were primiparous and $29(9.3 \%)$ had a history of previous abortion. Of the 43 GBS carriers, 37 (86\%) had no risk
Table 2. Occurrence of risk factors for GBS carriage during pregnancy, with results of vaginorectal screening for GBS and level of significance, in 310 women from Shiraz, Iran

\begin{tabular}{llcl}
\hline Risk factor & $\begin{array}{l}\text { GBS } \\
\text { carrier } \\
(\mathrm{n}=43)\end{array}$ & $\begin{array}{l}\text { GBS- } \\
\text { negative } \\
(\mathrm{n}=267)\end{array}$ & $\begin{array}{l}\mathrm{p} \\
\text { value }\end{array}$ \\
\hline $\begin{array}{l}\text { Intrapartum fever }\left(>37.5^{\circ} \mathrm{C}\right) \\
\text { Premature rupture of membranes }\end{array}$ & 1 & 9 & 0.81 \\
$\begin{array}{l}\text { Preterm }<37 \text { weeks } \\
\text { At least one risk factor }\end{array}$ & 2 & 33 & 0.77 \\
$\quad \begin{array}{l}\text { Present } \\
\text { Absent }\end{array}$ & 6 & 8 & 0.53 \\
\hline
\end{tabular}

factor for GBS colonization (gestational age $<37$ weeks; premature rupture of membranes; intrapartum fever $>37.5^{\circ} \mathrm{C}$ ).

GBS carrier status was not significantly associated with any risk factor. There were no statistically significant differences between risk factors $(\mathrm{p}>0.05)$ in colonized and noncolonized women (table 2).

Of the 310 women, 16 (5.2\%) admitted to taking antibiotics during pregnancy while approximately $50 \%$ could not recall why they were treated and the name of the antibiotic they took. However, urinary infection was the most frequent reason given for taking antibiotics, and amoxicillin or cephalexin was commonly used. Only 1 of the 16 women that received oral antibiotics was a GBS carrier (table 3).

\section{Microbiological Study}

Three hundred and nine microorganisms were isolated; 278 (89.7\%) women had positive culture and the remaining 32 were negative. The microorganisms isolated from rectovaginal samples are listed in table 4; nine groups or genera were identified. These included Enterococcus: 78 (25.1\%), coagulase-negative staphylococci: 60 (19.4\%), Escherichia coli: 46 (14.9\%), GBS: 43 (13.8\%), Candida: 33 (10.6\%), coagulase-positive staphylococci: 22 (7\%), Lactobacillus: 8 (2.6\%), streptococci other than GBS: 7 (2.6\%), Gardnerella: 7 (2.3\%), while Pseudomonas, Klebsiella and Proteus constituted less than $2 \%$ of the isolates. Gonococci and Haemophilus were sought, but not found. Overall, $89.6 \%$ of the women yielded significant cultures.

As for the order of microbial isolation, S. agalactiae (GBS) ranked 4th, with incidence of 43 (13.8\%) cases. From 50 streptococci isolated, 43 (86\%) were GBS. Also, 
Table 3. Antibiotics given orally at some time during pregnancy to 16 pregnant women and indication for treatment (self-reported by participating women by questionnaire)

\begin{tabular}{lc}
\hline Oral antibiotic therapy & $\mathrm{n}(\%)$ \\
\hline Antibiotics given & \\
$\quad$ Amoxicillin & $2(12.5)$ \\
Cephalexin & $6(37.5)$ \\
Erythromycin & $1(6.3)$ \\
Do not remember & $7(43.7)$ \\
Total & $16(100)$ \\
Indication for antibiotics & \\
UTI & $5(31.2)$ \\
URI & $2(12.5)$ \\
Do not remember & $9(56.3)$ \\
Total & $16(100)$ \\
\hline
\end{tabular}

$\mathrm{URI}=$ Upper respiratory tract infection; UTI = urinary tract infection.

from 82 staphylococcal isolates, 22 (26.8\%) were Staphylococcus aureus.

\section{Neonatal Population Characteristics}

There were 311 infants born to 310 women, of whom $170(54.5 \%)$ were female and the remaining 141 (45.3\%) male. The mean birth weight was 2,750 g. At 12 weeks after birth, only 1 neonate with early-onset infection was hospitalized due to poor feeding and lethargy. Of the blood, urine and CSF cultures performed, only CSF culture and Gram staining were positive for GBS.

\section{Discussion}

Nine groups or genera of microorganisms were identified in this study. The isolation of Enterococcus species and $E$. coli from the vagina is not usual [20-23]. It is known that antibiotics may modify the vaginal flora, but most of the women in this study had had no antenatal care. They were admitted in labor, and only $5.2 \%$ had received antibiotics that might have affected the vaginal flora. The presence of many of the organisms which are common in the rectum is most probably due to poor general hygiene.

The absence of lactobacilli from among most isolates in this study is also unusual, although it is known that lactobacilli are less frequently isolated elsewhere from women with disease of the lower genital tract [21-24].
Table 4. Microorganisms isolated from rectovaginal samples of pregnant women prior to labor in Shiraz, Iran

\begin{tabular}{lc}
\hline Organisms & $\begin{array}{l}\text { Isolated organisms } \\
\mathrm{n}(\%)\end{array}$ \\
\hline Streptococcus spp. & $50(16.7)$ \\
Streptococcus group B & $43(13.8)$ \\
Staphylococcus spp. & $82(26.4)$ \\
Staphylococcus aureus & $22(7)$ \\
Enterobacteriaceae & $48(15.6)$ \\
Escherichia coli & $46(14.9)$ \\
Klebsiella & $1(0.3)$ \\
Proteus & $1(0.3)$ \\
Enterococcus spp. & $78(25.1)$ \\
Candida spp. & $33(10.6)$ \\
Lactobacillus spp. & $8(2.6)$ \\
Gardnerella vaginalis & $7(2.3)$ \\
Pseudomonas & $3(1.0)$ \\
Total number of isolates per case & 1.1 \\
\hline
\end{tabular}

One explanation for this may be the $\mathrm{pH}$ of the vagina, which is known to become alkaline in certain morbid states. Isolation of Streptococcus and Staphylococcus species in this study is not surprising as these organisms are well-known etiological agents of puerperal sepsis. In recent years, there have been many reports on the possible role of Bacteroides species and related organisms in gynecological and obstetric infections. In the present study, Bacteroides species were not isolated by means of the routine anaerobic method used. It seems surprising that colonization of the lower genital tract with anaerobes has not arisen in this study. As the clinical laboratories are poor in the isolation of anaerobes and also the simple anaerobic culture used in our study did not yield any anaerobic bacteria including Bacteroides, a more detailed investigation of the possible role of anaerobic organisms in genital tract infections in this geographical locality is desirable. The frequency of Candida species in this study was lower than the findings of other researchers $[25,26]$. The association of Candida albicans with disease of the lower genital tract in pregnancy is well known.

Perhaps the most important fact that has emerged from this work is that the lower genital tract of most women in Shiraz at the time of labor is heavily colonized by pathogens. In order to prevent genital tract sepsis, therefore, labor should not be prolonged, and unnecessary trauma to the genital tract at the time of delivery should be avoided. 
The carriage rate of GBS in pregnant women in Shiraz, Iran was $13.8 \%$, which is similar to that seen in Kuwait [13] and Saudi Arabia [8], but considerably higher than in India [8]. Some authors also found a GBS isolation rate of $5-25 \%$ among Iranian parturient women in other parts of Iran $[16,17]$. Although our result shows a carriage rate within the aforementioned range, different methodology and geographical or socioeconomic conditions may be the reason for variations seen in GBS carriage rates.

By analysis of risk factors and carriage rates it is estimated that a maximum of $11.9 \%$ of women in this study would have been treated with prophylactic antibiotics due to prolonged rupture of membranes and none was under the screening approach of the CDC guidelines [15]. The presence of risk factors did not correlate well with vaginorectal carriage of GBS in this study. However, Oddie and Embleton [27] in a UK-based study did find a correlation between the presence of risk factors in mothers and the occurrence of GBS disease in their neonates, suggesting that analysis of larger groups of pregnant women may be required.

In our study, S. agalactiae was isolated from blood cultures of only 1 newborn, which contracted early-onset infection and was born to a GBS-positive mother. According to our knowledge, this case was a rare report of septicemia in infants due to GBS in Iran. This rate of infection is the same as the published rate of 5-10 per 1,000 colonized mothers. Such an attack rate is consistent with the literature [15]. One major limitation of this study is that a majority of the women could not recall if they were treated with antibiotics, hence education of the pregnant woman or the wording of the questionnaire may need to be improved.

\section{Conclusion}

This study revealed that $13.8 \%$ of pregnant women were colonized vaginorectally with GBS in Shiraz, Iran. There was a poor correlation between risk factors and GBS carriage. Potential future work in Iran should focus on large multicenter carriage studies of GBS in pregnant women, examination of culture versus rapid detection methods for identifying GBS carriage, and randomized controlled trials of strategies for prevention of neonatal GBS infection.

\section{Acknowledgments}

This work was financially supported by Shiraz University research grant and Shiraz University of Medical Sciences research grant No. 86-3675. The authors are grateful to Mr. N. Rafaatpour and Mrs. H. Asefi from Shooshtari Hospital for their helpful assistance.

\section{References}

- 1 Martins ER, Pessanha MA, Ramirez M: Analysis of group B streptococcal isolates from infants and pregnant women in Portugal revealing two lineages with enhanced invasiveness. J Clin Microbiol 2007;45:224229.

2 Holt DE, Halket J, Louvois D, Harvey D: Neonatal meningitis in England and Wales: 10 years on. Arch Dis Child 2001;84:85-89.

$\checkmark 3$ Vaciloto E, Richtmann R, de Paula Fiod Costa $\mathrm{H}$, Kusano EJ, de Almeida MF, Amaro ER: A survey of the incidence of neonatal sepsis by group B Streptococcus during a decade in a Brazilian maternity hospital. Braz J Infect Dis 2002;6:55-62.

4 Beardsall K, Thompson MH, Mulla RJ: Neonatal group B streptococcal infection in South Bedfordshire1993-1998. Arch Dis Child Fetal Neonatal Ed 2000;82:205-207.

5 Shah V, Ohlsson A: Prevention of early-onset group B streptococcal infection in the newborn: systematic review and recommendations. CMAJ 2002;166:928-930.
6 Watase RA, Hirata KY, Reppun TS: Group B Streptococcus: Prenatal Detection. Diagnostic Laboratory Services. 1996. Available at: http://www.dlslab.com/dls/page_server/527 54E241DB7601BE171326D2D.

$>7$ Bergeron MG, Ke D, Ménard C, Picard FJ, Gagnon M, Bernier M, Ouellette M, Roy PH, Marcoux S, Fraser WD: Rapid detection of group B Streptococci in pregnant women at delivery. N Engl J Med 2000;343:175-179.

$\checkmark 8$ Recommendation statement from the Canadian Task Prevention of group B streptococcal infection in newborns: Force on Preventive Health Care. CMAJ 2002;166:928-930.

$\checkmark 9$ Simões JA, Alves VM, Fracalanzza EL, Soares de Camargo R, Mathias L, Pires Milanez HM, Brolazo EM: Phenotypical characteristics of group B streptococcus in parturients. Braz J Infect Dis 2007;11:261-265.

10 Polin RA, Parravicini E, Regan JA, Taeusch HW: Bacterial sepsis and meningitis; in Taeusch HW, Ballard RA, Gleason CA (eds): Avery's Diseases of the Newborn, ed 8. Philadelphia, Elsevier Saunders, 2005, pp 551577.
-11 Berner R, Ruess M, Bereswill S, Brandis M: Polymorphisms in the cell wall-spanning domain of the $C$ protein $B$-antigen in clinical Streptococcus agalactiae isolates are caused by genetic instability of repeating DNA sequences. Pediatr Res 2002;51:106-111.

12 Spaetgens R, De Bella K, Ma D, Robertson S, Mucenski M, Davies HD: Perinatal antibiotic usage and changes in colonization and resistance rates of group $B$ streptococcus and other pathogens. Obstet Gynecol J 2002;100: 525-533.

13 Al-Sweih N, Hammoud M, Al-Shimmiri M: Serotype distribution and mother-to-baby transmission rate of Streptococcus agalactiae among expectant mothers in Kuwait. Arch Gynecol Obstet 2005;272:131-135.

14 Remington JS, Klein J (eds): Infectious Diseases of the Fetus and Newborn Infants, ed 5. Philadelphia, Saunders, 2001, pp 10911156. 
15 Schrag S, Gorwitz R, Fultz-Butts K, Schuchat A: Prevention of perinatal group B streptococcal disease. Revised guidelines from CDC. MMWR Recomm Rep 2002;51:1-22.

16 Rabiee S, Arab M, Yousefi Mashouf R: Epidemiologic pattern of vaginal colonization by group B Streptococcus in pregnant women in Hamadan, Central West of Iran. Iran J Med Sci 2006;31:106-108.

17 Nahaei MR, Ghandchilar N, Bilan N, Ghahramani P: Maternal carriage and neonatal colonization of Streptococcus agalactiae in Tabriz, Northwest Iran. Iran J Med Sci 2007; 32:181.

18 Trijbels-Smeulders M, Jonge GA, Pasker-de Jong PC, Gerards LJ, Adriaanse AH, Lingen RA: Epidemiology of neonatal group B streptococcal disease in the Netherlands before and after introduction of guidelines for prevention. Arch Dis Child Fetal Neonatal Ed 2007;92:271-276.
19 Christie R, Atkins NE, Munch-Peterson E: A note on lytic phenomenon shown by group B streptococci. Aust J Exp Biol Med Sci 1994; 22:197-200.

20 Altoparlak U, Kadanali A, Kadanali S: Genital flora in pregnancy and its association with group B streptococcal colonization. Int J Gynaecol Obstet 2004;87:245-246.

21 Simões JA, Giraldo PC: Prevalence of cervicovaginal infections during gestation and accuracy of clinical diagnosis. Infect $\mathrm{Dis} \mathrm{Ob}$ stet Gynecol 1998;6:129-133.

22 Balaka B, Agbèrè AD, Baeta $S$, Kessie K, Assimadi K: Bacterial flora in the genital tract the last trimester of pregnancy. J Gynecol Obstet Biol Reprod 2003;32:555-561.

23 Hay PE, Lamont RF, Robinson T, Morgan DJ, Ison $\mathrm{D}$, Pearson J: Abnormal bacterial colonisation of the genital tract and subsequent preterm delivery and late miscarriage. BMJ 1994;308:295-298.
24 Germain M, Krohn MA, Hillier SL, Eschenbach DA: Genital flora in pregnancy and its association with intrauteric growth retardation. J Clin Microbiol 1994;32:2162-2168.

25 Colli E, Bertulessi C, Landoni M, Parazzini F: Bacterial vaginosis in pregnancy and preterm birth: evidence from the literature. J Int Med Res 1996;24:317-324.

26 Regan JA, Klebanoff MA, Nugent RP: The epidemiology of group B streptococcal colonization in pregnancy, vaginal infections and prematurity study group. Obstet Gynecol 1991;77:604-610.

27 Oddie S, Embleton ND: Risk factors for early onset neonatal group B streptococcal sepsis: case-control study. BMJ 2002;325:308. 